\title{
Auditory Thalamus and Auditory Cortex Are Equally Modulated by Context during Flexible Categorization of Sounds
}

\author{
Santiago Jaramillo, Katharine Borges, and Anthony M. Zador \\ Cold Spring Harbor Laboratory, Cold Spring Harbor, New York 11724
}

In a dynamic world, animals must adapt rapidly to changes in the meaning of environmental cues. Such changes can influence the neural representation of sensory stimuli. Previous studies have shown that associating a stimulus with a reward or punishment can modulate neural activity in the auditory cortex (AC) and its thalamic input, the medial geniculate body (MGB). However, it is not known whether changes in stimulus-action associations alone can also modulate neural responses in these areas. We designed a categorization task for rats in which the boundary that separated low- from high-frequency sounds varied several times within a behavioral session, thus allowing us to manipulate the action associated with some sounds without changing the associated reward. We developed a computational model that accounted for the rats' performance and compared predictions from this model with sound-evoked responses from single neurons in $\mathrm{AC}$ and MGB in animals performing this task. We found that the responses of $15 \%$ of AC neurons and $16 \%$ of MGB neurons were modulated by changes in stimulus-action association and that the magnitude of the modulation was comparable between the two brain areas. Our results suggest that the $\mathrm{AC}$ and thalamus play only a limited role in mediating changes in associations between acoustic stimuli and behavioral responses.

Key words: auditory; categorization; flexibility; reward; stimulus-action association

\section{Introduction}

Categorization is the process by which potentially discriminable stimuli are assigned to functionally equivalent classes (Russ et al., 2007; Holt and Lotto, 2010). In a dynamic world, category definitions can vary, requiring organisms to adapt and switch rapidly between categorization rules depending on context. As a consequence of these changes, sensory stimuli that initially belong to one category and are associated with a particular behavioral response may, at different points in time, belong to other categories and require different responses. An impaired ability to adapt to these varying contingencies is one of the hallmarks of disorders such as schizophrenia and autism (Goldberg and Weinberger, 1988; Hill, 2004; Floresco et al., 2009).

Do sensory areas such as auditory thalamus and auditory cortex (AC) play a key role in flexible categorization or do they simply represent stimuli independently of their meaning? Previ-

Received Nov. 20, 2013; revised March 4, 2014; accepted March 8, 2014.

Author contributions: S.J. and A.Z. designed research; S.J. and K.B. performed research; S.J. and K.B. analyzed data; S.J., K.B., and A.Z. wrote the paper.

This work was supported by the National Institutes of Health (Grant 5R01DC012565-02) and the Swartz Foundation. We thank B. J. Burbach for technical assistance.

The authors declare no competing financial interests.

Correspondence should be addressed to Santiago Jaramillo, Department of Biology, 1210 University of Oregon, Eugene, 0R 97403. E-mail: sjara@uoregon.edu.

S. Jaramillo's present address: Institute of Neuroscience and Department of Biology, University of Oregon, Eugene OR 97403.

K. Borges's present address: Department of Physiology, Northwestern University, Chicago IL 60611.

DOI:10.1523/JNEUROSCI.4888-13.2014

Copyright $\odot 2014$ the authors $\quad 0270-6474 / 14 / 345291-11 \$ 15.00 / 0$ ous studies have demonstrated the formation of specific plasticity in auditory circuits when sounds are associated with the presence of a reinforcer (Weinberger, 1993; Fritz et al., 2003; Ohl and Scheich, 2005). It is not clear, however, if similar changes in representations occur when the sound-reinforcer association remains constant (e.g., if the sound always indicates the location of reward) but the sound-action association (e.g., the associated reward location) varies. Moreover, it is not known whether contextual influences on sound representation differ between AC and thalamus.

To address these questions, we characterized the soundevoked responses of neurons from the $\mathrm{AC}$ and its thalamic input, the medial geniculate body (MGB), during a task in which the stimulus-reward association was kept constant while the stimulus-action associations changed. Specifically, we designed a sound-categorization task for rats in which the boundary that separates low- from high-frequency sounds varied from one block of trials to the next several times within a behavioral session. Therefore, the action required to obtain reward for stimuli near the boundary changed from one contingency to another, whereas actions for stimuli far from the boundary remained unchanged.

We developed a computational model that accounted for the dynamics of the rats' choices as they switched between categorization contingencies. To test the role of the MGB and the AC under the assumptions of this model, we quantified (via extracellular recordings) the sound-evoked responses from neurons in these areas as animals switched between categorization contin- 
Table 1. Number of training sessions

\begin{tabular}{llll}
\hline Test & Rats & Sessions before testing & Average trials per session \\
\hline Behavior only & 9 & Mean $=87(\min =87)$ & $439 \pm 110$ \\
Electrophysiology & 8 & Mean $=201(\min =76)$ & $565 \pm 117$ \\
\hline
\end{tabular}

gencies. We found that, although subsets of sound-responsive cells were modulated between contingencies in each brain region, most neurons behaved as purely sensory neurons, consistent with the early stages in the model. These observations suggest that changing the stimulus-action association alone does not result in large changes in the representation of such stimuli in the auditory thalamus and cortex, as has been observed when the amount of reward or punishment predicted by each stimulus changes. Surprisingly, we found no differences in the features of evoked responses, number of modulated cells, or magnitude of modulation between thalamus and cortex.

\section{Materials and Methods}

Animal subjects. Animal procedures were approved by the Cold Spring Harbor Laboratory Animal Care and Use Committee and performed in accordance with National Institutes of Health standards. A total of 17 adult male Long-Evans rats (Taconic Farms) were used for analysis of switching behavior (eight of these were used for electrophysiological recordings). Rats had ad libitum access to food, but water was restricted. Free water was provided on days with no experimental sessions. Experiments were conducted in single-walled sound booths (Industrial Acoustics).

Behavioral task. Rats initiated each trial by poking their noses into the center port of a three-port chamber. After a silent delay of random duration (250-350 ms, uniformly distributed), a narrow-band sound was presented for $100 \mathrm{~ms}$. Animals were required to stay in the center port until the end of the sound and choose one of the two side ports for reward ( $24 \mu \mathrm{l}$ of water) according to the frequency of the sound (low-frequency: left port; high-frequency: right port). If animals withdrew before the end of the stimulus, the trial was aborted and ignored in the analysis.

Stimuli were chords composed of 16 simultaneous pure tones logarithmically spaced in the range $f / 1.2$ to $f \times 1.2$ for a given center frequency $f$. The intensity of the sounds was variable during initial training (50-70 $\mathrm{dB}-\mathrm{SPL}$ ), but fixed during testing at $60 \mathrm{~dB}-\mathrm{SPL}$. Animals were trained to discriminate between sounds centered at 6.5 and $14.2 \mathrm{kHz}$ in one contingency and 14.2 and $31 \mathrm{kHz}$ in another contingency. These frequencies are abbreviated herein as 6,14 , and $31 \mathrm{kHz}$. A single session consisted of several blocks of 300 trials (during behavioral training) or 150 trials (during electrophysiological recordings). The categorization contingency changed from one block to the next without any cue indicating the change except for the sound-reward relations. The initial contingency in a session was randomized from one day to the next. Measurements (behavioral or electrophysiological) were done only after animals had been extensively trained in the switching task (Table 1). Although, on average, electrophysiology animals were trained for more sessions than those used only for behavior, both cohorts showed similar performance levels $(82 \%$ and $84 \%$, respectively), suggesting that the number of sessions was sufficient to achieve an asymptotic level of performance. Animals for electrophysiological recordings were chosen in part because they provided more trials per session.

Analysis of behavioral performance. Data were analyzed using in-house software developed in Python (www.python.org). To illustrate the dynamics of performance after a switch in contingencies (see Fig. $2 A-C$ ), a first cohort of 12 animals was used. Trials from sessions starting with the low-boundary block were averaged to calculate the fraction correct for each trial number. Only sessions with 400 or more trials were included, resulting in 58 sessions per animal on average. The number of trials to switch for each animal (see Fig. 2D) was calculated from sessions starting with the low-boundary block, as the first trial for which the animal's average performance was above chance level (50\%) for two consecutive trials after the first switch.
Psychometric curves of 9 rats were estimated from sessions in which $10 \%$ of the trials contained sounds with center frequency logarithmically spaced between 6.5 and $31 \mathrm{kHz}$. The category boundary, which defined the associated reward port for each of these sounds, was set to the geometric mean of the training exemplars in each condition and the number of trials was balanced so that the fraction of rewards was the same on either side port. Psychometric curves were fit using Psignifit 3.0 (in the Python programming language). Briefly, a constrained maximum likelihood method was used to fit a logistic function with 4 parameters: $\alpha$ (the $50 \%$ threshold, or boundary), $1 / \beta$ (the slope of the curve), $\gamma$ (the lower asymptote), and $\lambda$ (the higher asymptote) as follows:

$$
\Psi(x)=\gamma+(1-\gamma-\lambda) \frac{1}{1+\exp (-g(x))} \quad g(x)=\frac{x-\alpha}{\beta}
$$

Computational learning model. We implemented a firing rate model with plastic synapses. Each input $\left(x_{\mathrm{i}}\right)$ corresponded to a different soundfrequency channel and the output $(z)$ provided a binary signal that indicated a leftward or rightward choice. For a given stimulus, the value of each input $\left(x_{\mathrm{i}}\right)$ was calculated according to a Gaussian receptive field $(\mathrm{RF})$ in logarithmic space as follows:

$$
x_{i}=e^{-\frac{\left(f-f_{i}\right)^{2}}{2 \sigma^{2}}}
$$

where $f$ and $f_{\mathrm{i}}$ correspond to the logarithm of the frequency of the stimulus and the RF center, respectively. The width of the RF was set to $0.4 \log$ units and the RFs were logarithmically spaced between 5 and $40 \mathrm{kHz}$. The output on each trial was calculated as the sum of the inputs weighted by the synaptic strengths passed through a saturating nonlinearity $(S)$ as follows:

$$
z=S(\vec{w} \cdot \vec{x})=S\left(\sum_{i} w_{i} x_{i}\right)
$$

The synaptic strengths $\left(w_{\mathrm{i}}\right)$ could take positive or negative values and were modified according to the outcome of the previous trial using a perceptron learning rule (Bishop, 1995) as follows:

$$
\vec{w}_{t+1}=\vec{w}_{t}+\eta\left(d_{t}-z_{t}\right) \vec{x}_{t}
$$

where $d_{\mathrm{t}}$ was the desired choice for trial $t$ and $\eta$ was the learning rate of the system. For the examples in Fig. 4, $\eta$ was fit to replicate the average switching speed of the rats, resulting in a value of 0.03 .

Noise was introduced at only one stage (inputs, synaptic strengths, or outputs) on each simulation. For the inputs, values drawn from a Gaussian distribution were added to each $x_{\mathrm{i}}$. The SD of this noise was fit to replicate the average asymptotic performance of the rats $(80-90 \%)$, resulting in a value of 0.4 . For the synaptic strengths, Gaussian noise was added to each $w_{\mathrm{i}}$. An SD of 0.14 resulted in an asymptotic performance similar to that of the rats. For the outputs, Gaussian noise was added to the weighted sum of inputs before the nonlinearity. An SD of 1 replicated the average asymptotic performance of the rats.

Surgery. Animals were anesthetized with an intraperitoneal injection of a mixture of ketamine $(60 \mathrm{mg} / \mathrm{kg})$ and medetomidine $(0.5 \mathrm{mg} / \mathrm{kg})$. Wounds were infiltrated with lidocaine. Rats were surgically implanted with a custom-made microdrive in left AC (two rats), right AC (two rats), or the left auditory thalamus (four rats) containing eight independently adjustable tetrodes. For AC recordings, electrodes were implanted between 3.5 and $6 \mathrm{~mm}$ posterior to bregma and $6.5 \mathrm{~mm}$ left or right from the midline. For MGB recordings, electrodes were implanted between -5.6 and $-6 \mathrm{~mm}$ posterior to bregma and between 3.6 and $3.8 \mathrm{~mm}$ left from midline. For thalamic recordings, electrodes were lowered beyond 4 $\mathrm{mm}$ from the brain surface until we observed sound-evoked responses. Animals were allowed to recover for several days before resuming water restriction and starting recording sessions. Average performance during recordings sessions ( $86 \pm 6 \%$, mean $\pm \mathrm{SD}$ ) was comparable to the performance estimated for these animals before implantation $(84 \pm 4 \%)$.

Neuronal recordings. Each tetrode consisted of four polyimide-coated nichrome wires (diameter $12.7 \mu \mathrm{m}$; Kanthal Palm Coast) twisted together 
A

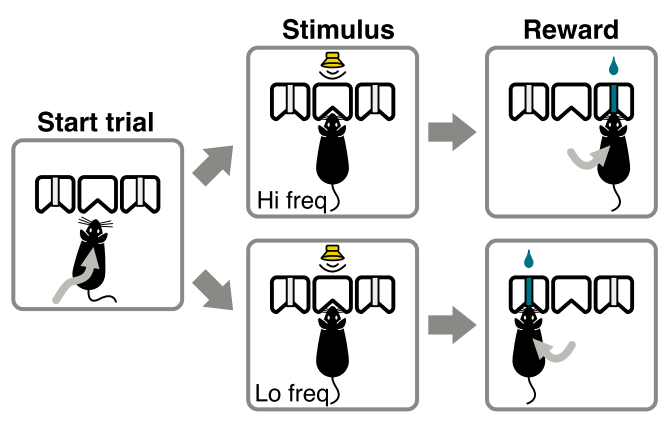

B

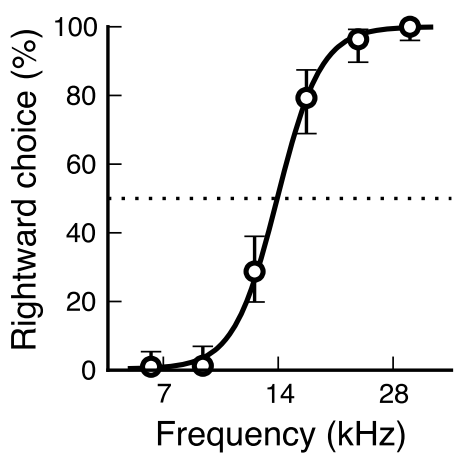

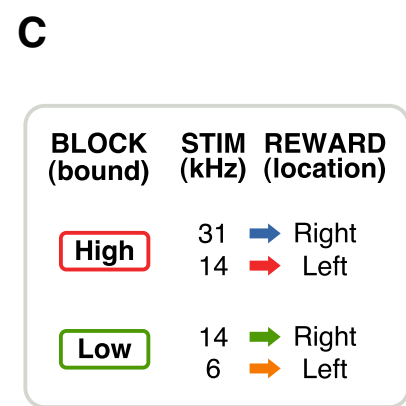

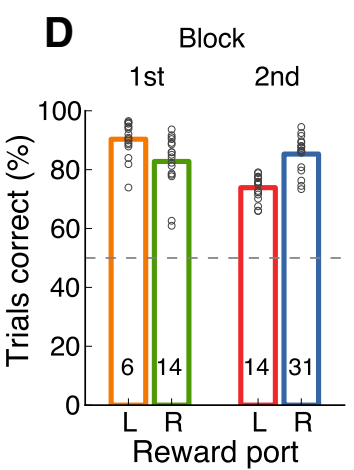

Figure 1. Flexible sound-categorization task. $\boldsymbol{A}$, Rats initiated each trial by poking their noses into the center port of a three-port chamber. A narrow-band sound was presented for $100 \mathrm{~ms}$, indicating the location of reward: left for low-frequency sounds and right for high-frequency sounds. $\boldsymbol{B}$, Psychometric performance for one rat during one discrimination session demonstrating that animals can achieve perfect performance on easy discriminations ( 6 vs 31 kHz; error bars are $95 \%$ confidence intervals). C, To introduce flexibility in the task, each session consisted of alternating blocks of 300 trials: in one block type, rats were required to discriminate between 31 and $14 \mathrm{kHz}$; in the other block type, between 14 and $6 \mathrm{kHz}$. This was equivalent to setting the category boundary to either a "high" or a "low" frequency value. The middle frequency sound ( $14 \mathrm{kHz}$ ) changed its meaning from one block type to the other. $D$, Average performance for each of the 17 rats on the first and second blocks of trials when the task started on the low-boundary block. Each circle corresponds to the average performance across multiple sessions for one animal for each stimulus, ignoring the first 100 trials after a block switch. Chance level was 50\%. E, Same as D, but when the task started on a high-boundary block. Performance level was above chance in all conditions, but consistently lower for the reversing stimulus (14 kHz).

and gold plated to an impedance of $0.3-0.4 \mathrm{M} \Omega$ at $1 \mathrm{kHz}$. Electrical signals passed through unity-gain head stages (Neuralynx) connected to a custombuilt array of tetrode microdrives before reaching the acquisition system. Signals were recorded using a Neuralynx Cheetah 32 recording system. Tetrode depths were adjusted at lest $12 \mathrm{~h}$ before recording sessions to sample an independent population of cells each time while maintaining a stable recording throughout the session. Tetrode locations were confirmed histologically based on electrolytic lesions and fluorescent markers (electrodes were coated with DiI before implanting). Track reconstructions indicated that we sampled mostly auditory cortical neurons from the primary field (A1; see Fig. $5 A$ ), although we cannot rule out that some neurons were located in the posterior field (Doron et al., 2002). Thalamic recordings targeted all subnuclei (dorsal, ventral, and medial) of the MGB (see Fig. 5B).

Analysis of neuronal data. Data were analyzed using in-house software developed in Python (www.python.org). Spiking activity of a total of 665 single cells from the AC and 217 from the auditory thalamus was isolated by a combination of an automated expectation maximization algorithm (Klustakwik; Kadir et al., 2013) and by manually clustering spike shapes using in-house software. Our analysis focused on cells that showed changes in activity in response to the middle (reversing) frequency, resulting in a set of 191 cortical cells and 57 thalamic cells. Only cells for which we had at least three blocks of 150 trials per block (two switches) were included. To evaluate responsiveness to each frequency, we quantified spike counts in nonoverlapping bins of $25 \mathrm{~ms}$. We used these values to calculate $z$-scores for each bin with respect to the bin starting $100 \mathrm{~ms}$ before sound onset. We considered a cell responsive if the $z$-score of any bin during the response period $(0-150 \mathrm{~ms})$ fell outside the range $(-3,3)$.

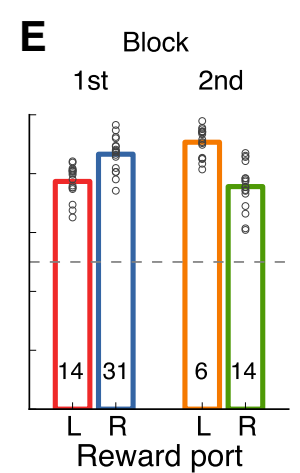

For each responsive cell, we calculated a modulation index (MI) to quantify changes in evoked response to the same stimulus between categorization contingencies as follows:

$$
\mathrm{MI}=\frac{r_{c}-r_{i}}{r_{c}+r_{i}}
$$

where $r_{\mathrm{c}}$ and $r_{\mathrm{i}}$ correspond to the evoked firing rates when the stimulus indicated reward on the contralateral or ipsilateral side from the recording site. We included only correct trials in this calculation. We tested statistical significance of the modulation for each cell via a rank-sum test between the evoked firing on each contingency (at a significance level of 0.05). To exclude effects of nonstationarity, cells were counted as significantly modulated only if the sign of the modulation flipped from one contingency switch to the next. That is, if the firing rate increased after the first contingency switch (when the meaning of the sound changed from left reward to right reward), then the firing rate must decrease after the second switch (when the sound indicated again left rewards).

Ideal observer analysis. For the ideal observer analysis shown in Figures 7 and 8, we counted the number of evoked spikes in the range $10-$ $150 \mathrm{~ms}$ from sound onset for each cell on each trial. We then estimated the distribution of these spike counts for each stimulus-action condition from half of the trials (randomly selected) and used samples from this distribution to train a linear discriminator (Machine Learning Python; Albanese et al., 2012). We used the other half of the trials to estimate the discrimination accuracy of the observer given each neuron on each condition. We quantified the sound discrimination performance (see Fig. 7) independently on each contingency, resulting in two performance values for each neuron. For choice discrimination, we separated all trials with associated left rewards from those associated with right rewards (independently of the stimulus presented). These two groups defined the dataset given to the linear discriminator.

To evaluate whether changes in evoked response between contingencies improved discrimination, we measured the performance of the observer with a modified dataset in which the trials containing the reversing frequency were swapped between contingencies. For example, instead of discriminating between evoked responses from $6 \mathrm{kHz}=\mathrm{L}$ and $14 \mathrm{kHz}=$ $\mathrm{R}$ (both from the low-boundary condition), the observer had to discriminate between responses from $6 \mathrm{kHz}=\mathrm{L}$ and $14 \mathrm{kHz}=\mathrm{L}$ (from the lowand the high-boundary conditions, respectively).

\section{Results}

Rats reliably switched between categorization contingencies

To study the role of AC and MGB during flexible categorization of sounds, we developed a frequency discrimination task (Fig. $1 A, B)$ in which the boundary that separates low- from highfrequency sounds varied several times within a behavioral session (Fig. 1C). Rats initiated each trial by poking their noses into the center port of a three-port chamber. A narrow-band sound (chord) was presented for $100 \mathrm{~ms}$, indicating the location of reward: left for a low-frequency sound and right for a highfrequency sound. Animals were tested in blocks of trials: in the low-boundary block, animals were required to discriminate between sounds centered at 6 and $14 \mathrm{kHz}$; in the high-boundary 
block, they were required to discriminate between 14 and $31 \mathrm{kHz}$. As a result, the sound at $14 \mathrm{kHz}$ belonged to the highfrequency category in one block of trials and to the low-frequency category in the next block and the animal was required to change its behavioral response to this sound to successfully collect reward. We refer to this sound as the reversing stimulus. Within a session, animals switched back and forth between these two contingencies every 300 trials, without any additional cue in addition to the stimuli and rewards. All trained rats performed the task accurately and showed higher performance in response to nonreversing stimuli (6 and $31 \mathrm{kHz}$ ) compared with the reversing stimulus $(14 \mathrm{kHz})$, even 100 trials after a change in contingency $(p<$ 0.01 , Wilcoxon signed-rank test; Fig. $1 D, E)$. Average performance across conditions for animals selected for electrophysiological recordings $(84 \pm 4 \%$, mean $\pm \mathrm{SD})$ was comparable to the performance of all other animals in the study ( $82 \pm 4 \%)$.

Rats adapted rapidly after changes in category definitions (Fig. 2). For most animals, 12 trials were sufficient to cross the $50 \%$ performance level after a stimulus changed its meaning (Fig. 2D). Surprisingly, changes in accuracy occurred not only for stimuli that changed association between contingencies, but also for stimuli that never changed their associated reward port (Fig. 2C, yellow arrows). This effect was consistent across all animals $(p<0.01$, Wilcoxon signed-rank test; Fig. $2 E)$. We hypothesized that these effects were present because animals' choices depended on the distance between the perceived stimulus and their internal categorization boundary. As a result, boundary shifts after a switch affected performance for all stimuli (Fig. $2 F$ ).

To quantify the animals' internal category boundary, we tested performance in response to sounds with frequencies different from the three exemplars used during training. Rats generalized to other stimuli in a monotonic fashion (Fig. 3A) and a shift in the internal categorization boundary was evident for all animals as they switched from one contingency to another $(p<0.01$, Wilcoxon signed-rank test; Fig. $3 B$ ) without apparent changes in the slope of the psychometric function ( $p=0.17$, Wilcoxon signed-rank test, data not shown).

These observations were consistent with the idea that choices depend on the distance between an internal categorization boundary and the perceived stimuli. During the high-boundary block, animals set their subjective boundary between 14 and $31 \mathrm{kHz}$. As animals switched to the low-
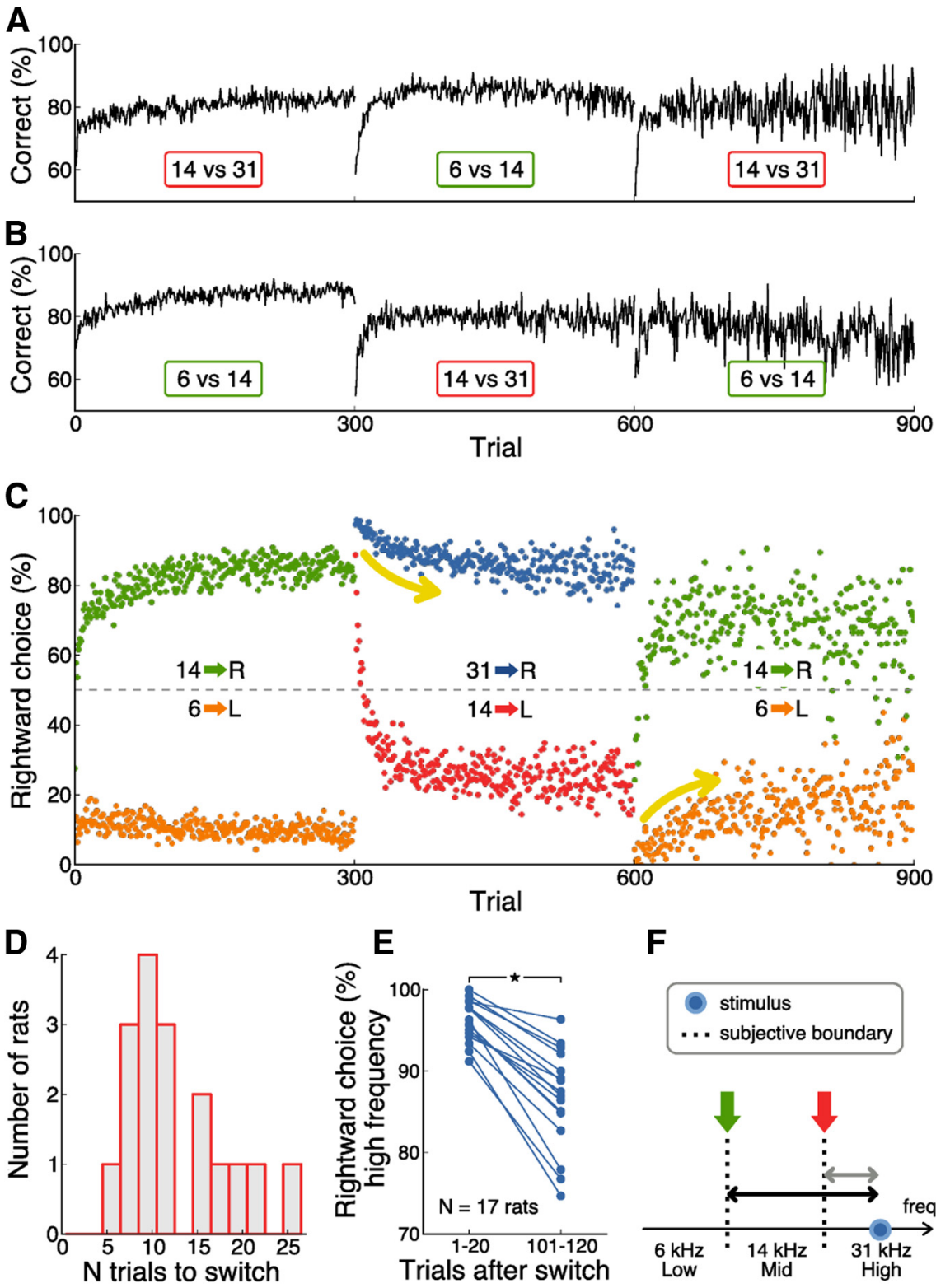

F

Figure 2. Switching contingencies affected responses to all stimuli. $A$, Average performance across sound-action associations for an initial cohort of 12 rats (trained in parallel) on sessions that started with the high-boundary block. Blocks were 300 trials long. Averages were calculated for each trial across sessions and animals without time averaging. The increased variability on the last trials is due to averaging across less number of samples because not all animals performed 900 trials. $\boldsymbol{B}$, Same as in $\boldsymbol{A}$, but for sessions that started with the low-boundary block. $\boldsymbol{C}$, Average performance across rats in $\boldsymbol{A}$ for each sound-action association. Each dot represents the percentage of rightward choices for one trial in a session taken across several sessions from each animal. This plot shows only sessions that started with the low-boundary block. Yellow arrows indicate how performance also changed for stimuli that never changed their associated category. Increased variability on the last trials is due to fewer samples, as explained in $\boldsymbol{A}$. $\boldsymbol{D}$, Number of trials required to switch sound-action associations for each of the 17 animals trained in the full task. The value plotted corresponds to the trial in which each animal crossed the $50 \%$ level of rightward choices for the reversing stimulus ( $14 \mathrm{kHz}$ ) after the first block switch. Most rats switched before 12 trials. $\boldsymbol{E}$, Change in behavioral responses for a sound that never changed association. Each dot is the percentage of rightward choices for each of the 17 animals in response to the $31 \mathrm{kHz}$ sound in trials $1-20$ or 101-120 after a switch. A decay in performance is evident for all rats. $\boldsymbol{F}$, lllustration of the shift in subjective category boundary that accounts for the decay in performance in $E$. At the end of the first block and the beginning of the second block, the boundary is located between 6 and $14 \mathrm{kHz}$ (green arrow). In this case, the distance between the boundary and 31 kHzis large and small noise will not change the location of the stimulus with respect to the boundary. As the subject is exposed to more trials from the second block, the boundary shifts between 14 and $31 \mathrm{kHz}$ (red arrow). Under this condition, noise in the representation of either the stimulus or the boundary may cause a flip in the relation between stimulus and boundary, causing a mistake in choice.

boundary block, the subjective boundary shifted away from the $31 \mathrm{kHz}$ sound, resulting in an improvement in performance for that stimulus. Switching back to the high-boundary block produced the opposite shift in the boundary and a reduction in per- 
A

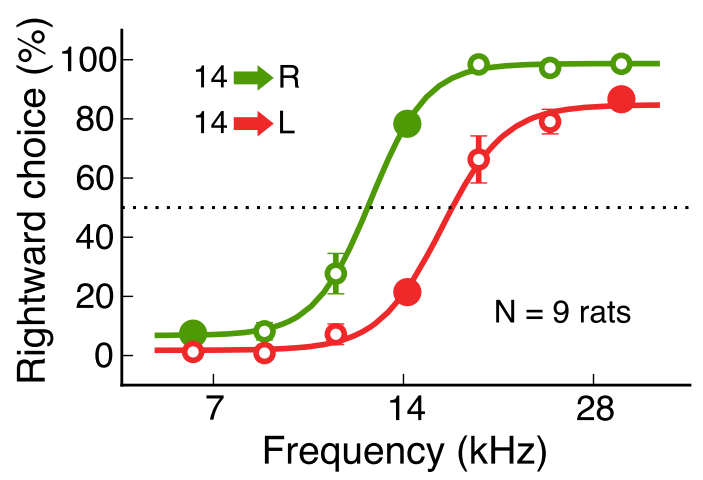

B

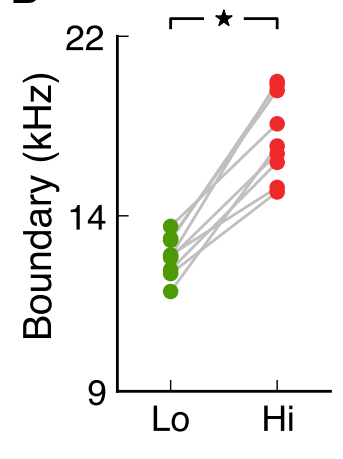

cution process reproduced the animals' asymptotic performance, but did not capture the dynamics of behavioral performance after a contingency switch (Fig. 4C).

These computational results provide an explanation for the paradoxical changes in performance for nonreversing stimuli. The model also demonstrates that the task can be solved without an explicit representation of the subjective category boundary in the firing rates of neurons. Last, the results of our simulations suggest that the animals' mistakes are due mostly to imperfect representation of either the stimuli or the internal categorization boundary, not to lack of understanding of the task rules or errors in choice execution.

This model assumes distinct physiological responses along the sensorimotor pathway. At early stages, neurons represent the sounds independently of their animals. Red and green correspond to estimates from the low-boundary and high-boundary blocks, respectively, from the nine rats tested. In $10 \%$ of trials (open circles), animals were probed with frequencies different from those in the training set (solid circles). Each circle indicates the average of rightward choices for each frequency across animals, ignoring the first 100 trials after a switch. Error bars indicate $S E$. Curves correspond to logistic fits given the choice for each trial. $\boldsymbol{B}$, All rats shifted their internal categorization boundary between blocks of trials. Each dot represents the estimated boundary from the logistic fit for each animal.

formance for the $31 \mathrm{kHz}$ sound. To explicitly test the predictions of this model when implemented in a neurally plausible fashion, we simulated an adaptive decision system and presented it with the same stimulus-action contingencies as were given to the animals.

\section{Adaptive system with imperfect sensory representations accounted for the dynamics of behavior}

A striking feature of the animals' behavior was that a contingency switch elicited paradoxical changes in performance for stimuli that never changed category (Fig. 2E). Instead of improving with time, performance for these stimuli decayed as animals were exposed to more trials in a new categorization contingency. As illustrated in Figure $2 F$, this effect is present if choices depend on the distance between the frequency of the stimulus and a subjective category boundary. One possible mechanism for implementing this computation would be an explicit representation of the boundary by the firing rate of some neurons. We hypothesized that a different mechanism could account for the observed dynamics of performance; that is, the boundary is represented by synaptic strengths between sensory and decision neurons. To test this hypothesis, we implemented a firing rate model in which synaptic strengths changed after the outcome of each trial (Fig. 4A).

A noiseless version of this model (data not shown) successfully learned to switch between categorization contingencies without requiring an explicit representation of the category boundary in the firing rate of neurons. However, the noiseless system quickly reached perfect discrimination performance on each block of trials, in contrast to the suboptimal performance achieved by the animals. We hypothesized that the performance level of our rats was due to imperfect representations of the following: (1) the sensory signals, (2) the categorization boundary, and (3) the neural signals representing the selected action, or a combination of these.

When Gaussian noise was added to the sensory representation, the model reproduced not only the rapid switches observed in performance dynamics, but also the paradoxical changes in accuracy for the nonreversing stimuli (Fig. 4B). Noise added to the synaptic strengths, which implicitly represent the subjective categorization boundary, also accounted for these behavioral features (data not shown). In contrast, added variability to the exe- meaning (Fig. 4D). At later stages, neurons can be sensitive to both the identity and the meaning of sounds (Fig. $4 E$ ). To test which of these possibilities best matches the activity of neurons in the AC and MGB, we recorded neural responses from animals performing the flexible categorization task.

\section{Subpopulations of neurons in AC and MGB are sensitive to context}

We quantified the sound-evoked responses of single neurons from the AC (Fig. 5A) and the MGB (Fig. 5B) as animals switched between categorization contingencies. The dynamics of evoked responses were similar between the $\mathrm{AC}$ and the $\mathrm{MGB}$, with both areas containing some neurons that responded by increasing their firing (Fig. $5 C, D$ ) and others that decreased their firing (Fig. $5 E, F)$. Neurons in both areas showed various diverse patterns of onset, sustained, and offset responses when presented with the $100 \mathrm{~ms}$ sounds of different frequencies during the task (Fig. $5 G, H)$. From the 217 cells recorded from the thalamus, $22 \%$ had increases in firing in the first $50 \mathrm{~ms}$ after a sound (onset), 12\% increases in firing in the next $50 \mathrm{~ms}$ (sustained), 13\% increases in firing in the $50 \mathrm{~ms}$ after sound offset, and $12 \%$ had a suppression in firing during sound presentation. Combinations were often found. From the 665 cortical cells, 19\% had onset increases, 15\% sustained, $18 \%$ offset, and $17 \%$ had a suppression in firing during sound presentation, many of them in combination. For the remainder of the analysis, we focused on neurons that showed responses to the reversing middle-frequency stimulus (Table 2).

We compared the responses evoked by the reversing middlefrequency stimulus $(14 \mathrm{kHz}$ ) between categorization contingencies. We found that neural responses (in the range 10-150 ms from sound onset) varied between contingencies in a subset of neurons from the AC (Fig. 6A) and from the auditory thalamus (Fig. 6B). Other neurons, in contrast, reliably represented the stimulus independent of the action associated with it (Fig. 6C,D). To exclude effects from nonstationarity, only cells that were modulated in opposite directions after consecutive switches in contingencies were counted as modulated. We reasoned that if evoked firing in the AC or MGB were correlated with the side of reward, then activity should be consistently higher for one of the two contingencies as animals switch back and forth between them. In the $\mathrm{AC}, 15 \%$ of the cells that responded to the reversing 
A

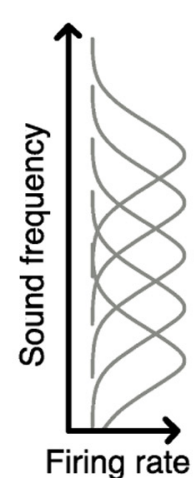

$$
\text { sensory }
$$

noise synaptic

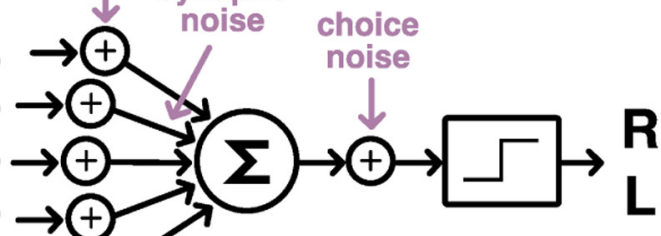

$\rightarrow \oplus$

1

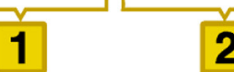

B
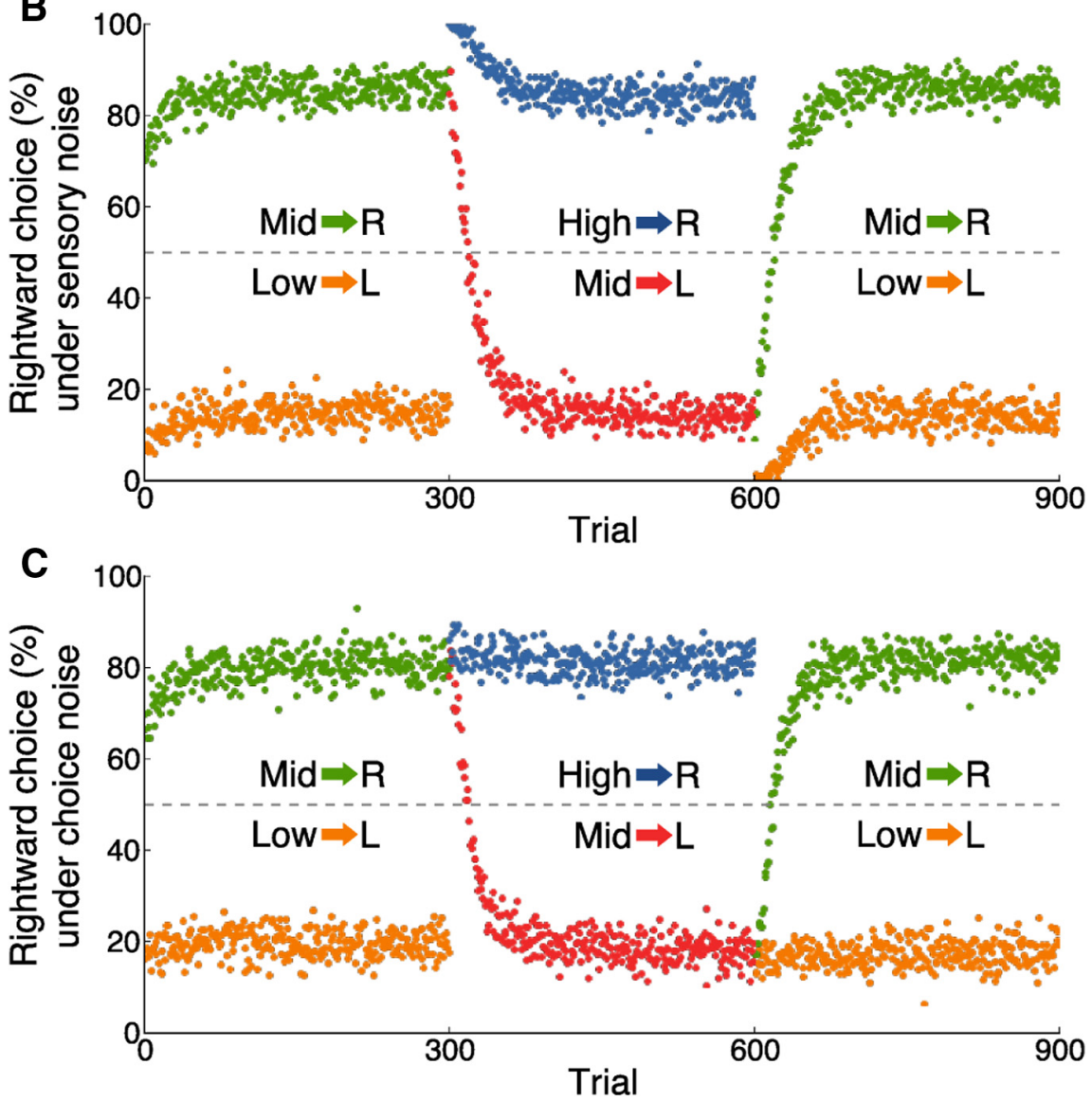

D
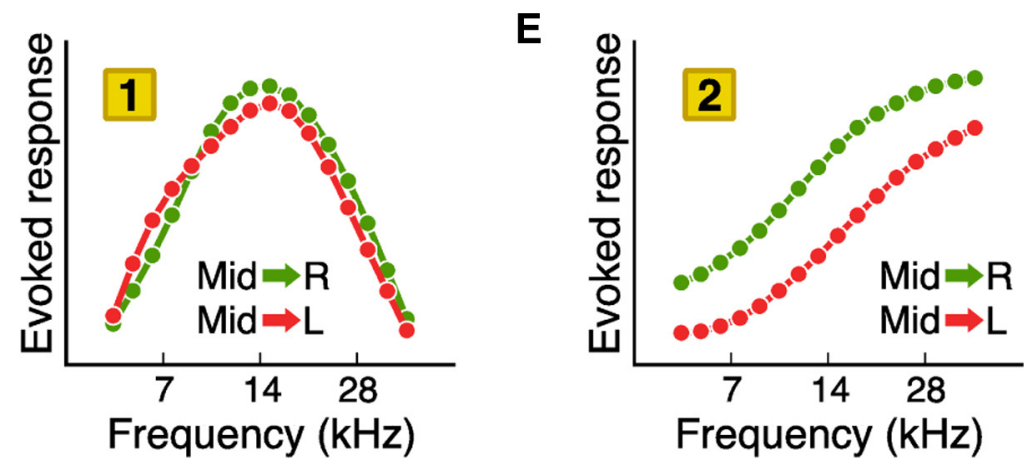

Figure 4. An adaptive system with noisy representations accounted for the dynamics of behavioral performance. $A$, Learning model. Yellow labels indicate each stage. Inputs (stage 1) correspond to frequency channels with imperfect (noisy) representations of the acoustic stimulus. A decision neuron (stage 2) sums these inputs, weighted by the strength of each synapse. These strengths change according to the outcome of each trial. The binary output indicates either a left or right choice. Noise was added at one of three locations: the sensory signals, the synaptic strengths, or the choice signal. $\boldsymbol{B}$, Average performance on each trial when noise stimulus (29/191) were significantly modulated ( $p<0.05$, rank-sum test) between contingencies (Fig. 6E). A similar subset of responsive cells, $16 \%$ (9/57) was significantly modulated in the auditory thalamus (Fig. 6F). An initial evaluation of changes in firing rate, which did not require the criterion of consistent modulation direction, yielded a slightly higher fraction of modulated cells: $22 \%$ in $\mathrm{AC}$ and $19 \%$ in MGB.

We also evaluated the modulation of baseline activity ( -150 to $-10 \mathrm{~ms}$ from sound onset) and found that only $4.2 \%$ of cells from AC and 5.3\% from MGB were modulated in both baseline and evoked firing. This indicates that only one-third of the cells with modulation in evoked response could be explained by changes in the baseline firing. In addition, evaluating the modulation in the range $10-100 \mathrm{~ms}$, which excludes any early movements of the animal but also offset responses, resulted in $10 \%$ of cells modulated in cortex and $17 \%$ in the thalamus. The modulation of evoked responses consisted mostly of a change in the amplitude of the response and did not affect the type of response pattern (onset, offset, or sustained) of neurons. We found no evidence of a systematic location of the modulated neurons in particular regions of AC (modulated cells were found at various depths on the same electrode) or MGB (modulated cells were found on electrodes located in different subnuclei). On average, the magnitude of the modulation was not different between cortical and thalamic cells ( $p=0.4$, Levene's test on the variance of the modulation index; Fig. $6 G$ ).

These results indicate that soundevoked responses in a small subset of neurons in the sensory pathway are modulated by the stimulus-action association. Most neurons, however, behaved like those in the early stages of processing from our model (Fig. 4D) and were not influenced by the associated action. Surprisingly, the observed modulation of re-

was added to the sensory signals. The noise parameter was chosen so that asymptotic performance for the extreme frequencies matched that of the rats. This model reproduced the decay in performance for the high frequency (in blue). C, Average performance when noise was added to the choice signals. This model did not account for the decay in performance after a contingency switch. $\boldsymbol{D}$, Simulated evoked responses for a sensory neuron (stage 1, tuned to the middle frequency) are similar between the two contingencies (green: low-boundary; red: high-boundary). $\boldsymbol{E}$, Simulated responses of the decision neuron (stage 2) depend on both the stimulus and its meaning. 

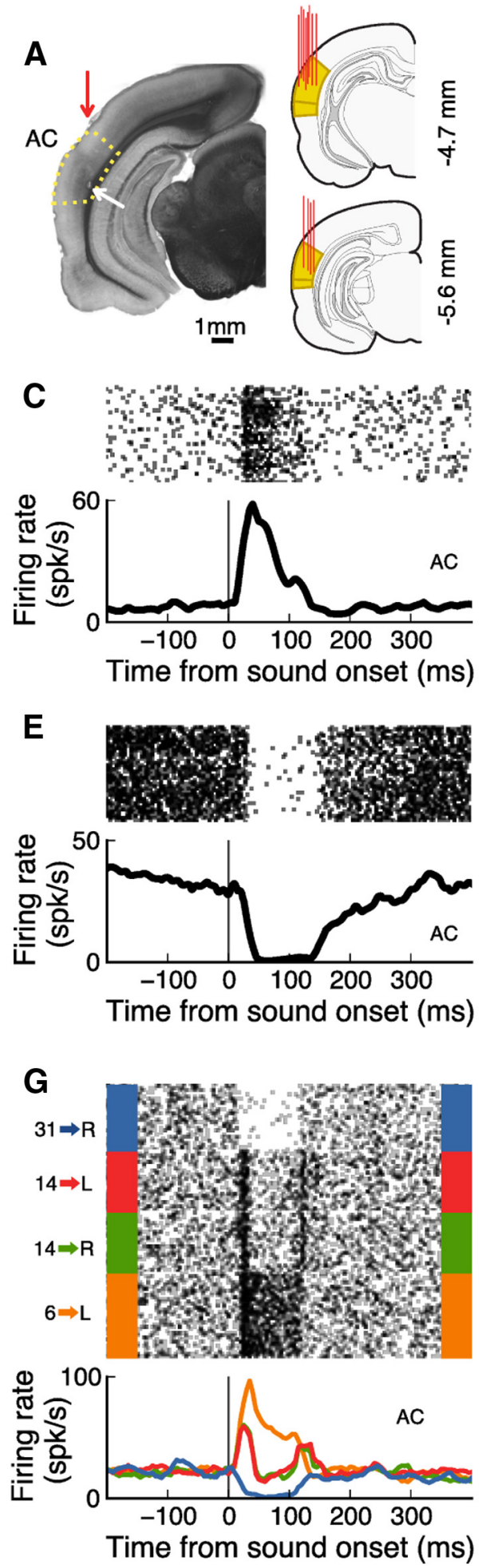

Figure 5. Physiological responses to sounds were similar between cortical and thalamic cells during the task. $A$, Reconstruction of electrode tracks from the AC. Left, Coronal slice showing an example track. The red arrow indicates where the electrode was inserted. The white arrow indicates the electrolytic lesion at the end the recordings. Right, Reconstruction of all electrodes (red) from all animals projected onto the closest of the coronal slices shown (projected onto the left hemisphere). The yellow area includes the primary and ventral fields of the AC (Doron et al., 2002). The number indicates the distance from bregma. $\boldsymbol{B}$, Same as $\boldsymbol{A}$, but for the thalamic recordings. The yellow area includes all subnuclei of the MGB (Paxinos and Watson, 2005). $\boldsymbol{C}$, Sound-evoked response of one cortical cell that increased its firing after the presentation of one of the target sounds. $\boldsymbol{D}$, Thalamic cell with a response similar to that in $\boldsymbol{C}$. $\boldsymbol{E}$, Example physiological response of one cortical cell that decreased its firing after the presentation of one of the target sounds. $\boldsymbol{F}$, Thalamic cell with a response similar to that in $\boldsymbol{E}$. $\boldsymbol{G}$, Response of a cortical cell to the target sounds in each of the four stimulus-action conditions. $\boldsymbol{H}$, Response of a thalamic cell in each condition. The same cell can show different response dynamics depending on sound frequency. sponses was equally strong in auditory thalamus and AC.

Modulation of responses did not improve the discriminability of sounds When solving tasks that require flexibility, the nervous system may use other strategies in addition to those presented in our model (Fig. 4) to improve performance. For example, top-down signals may influence the processing of sounds in sensory areas such that the discriminability of stimuli in each contingency is enhanced. Our simulations suggest that animals do not achieve perfect levels of performance due to imperfect neural representations of either the stimulus or the subjective category boundary. Given these psychophysical demands, we hypothesized that contextual modulation of neuronal responses in the sensory pathway may be playing a role in improving the discriminability of stimuli.

To test this "adaptive system" hypothesis, we first evaluated the ability of an ideal observer to discriminate between sounds within each block of trials separately, given the evoked responses of each neuron. Performance was quantified as the fraction of trials in which the observer correctly estimated which of the two sounds was presented. We found that the performance of the observer was similar for neurons in the AC and the auditory thalamus ( $p=0.21$, rank-sum test; Fig. $7 A$ ).

This hypothesis makes a specific prediction regarding the direction of modulation of activity for neurons tuned to either the high- or low-frequency sounds in our task: evoked responses to the reversing stimuli should vary so that the discriminability of 31 versus $14 \mathrm{kHz}$ in one block of trials and 14 versus $6 \mathrm{kHz}$ in the other block, increases (Fig. $7 C$, arrows). To determine whether our data were consistent with this prediction, we compared the performance of the ideal observer with that of an observer presented with the evoked responses after swapping the middle-frequency trials between blocks. The analysis was performed only on cells tuned to the high or low frequencies because the "adaptive system" hypothesis makes no predictions regarding the direction of modulation that would improve discrimination for neurons tuned to the reversing stimulus $(14 \mathrm{kHz})$; swapping trials would simply improve performance in one contingency while decreasing performance in the other.

If the modulation helped to improve performance, then the discrimination ability of the observer would decrease 
when trials were swapped (Fig. $7 D, E$ ). We found that this was not the case in either the AC ( $p=0.67$ for low-block; $p=0.98$ for high-block; $n=77$ ) or the auditory thalamus $(p=0.26$ for low-block; $p=0.8$ for high-block; $n=31$ ) and that, whereas some neurons behaved as predicted, a similar number of neurons were modulated in the opposite direction. These results did not change when analyzing only the contingencies in which performance was highest for each neuron. Likewise, the effect for neurons with high discrimination performance (Fig. $7 D, E$, top-right corner) was comparable to the effect on all other neurons. Results were also similar when analyzing only neurons with statistically significant modulation in the cortex $(p=$ 0.8 for low-block; $p=0.65$ for high-block; $n=10)$ or the thalamus $(n=2)$. The observed modulation of sound-evoked responses was therefore not consistent with an increase in discriminability for each context.

\section{Modulation of responses did not enhance the representation of choice} An alternative cause for the modulation of firing rates could have been that neurons in AC and MGB were affected by signals related to the animals' choices that were mediated, for example, by feedback from motor-related areas. To test this hypothesis, we evaluated the ability of an ideal observer to discriminate between right and left choices based on the evoked responses of each neuron. This ideal observer analysis tells us explicitly how neural activity is related to behavioral performance and therefore complements our quantification of statistically modulated cells (Fig. 6). This analysis includes all trials (correct or incorrect) grouped according to the animals' decisions. In contrast to Fig. $7 A$, this analysis includes the responses to low- and high-frequency stimuli ( 6 and 31 $\mathrm{kHz}$ ) in addition to the middle-frequency reversing stimulus $(14 \mathrm{kHz})$. The performance of the observer was similar for neurons in the $\mathrm{AC}$ and the auditory thalamus $(p=0.2$, rank-sum test; Fig. $8 A$ ).

Because the observer is exposed to trials with extreme-frequency sounds associated to only one reward side, choice discrimination could be explained partly by these signals. Nevertheless, this quantity allows us to evaluate whether modulations in the evoked response were consistent with a representation of choice. For neurons tuned to the middle frequency, this hypothesis predicts that evoked responses to sounds associated with left choices should be separable from those associated with right choices (Fig. $8 C$ ). Similar to the previous section, we compared performance of the observer with that calculated from evoked responses when swapping the middle-frequency trials between blocks (Fig. $8 D, E)$. If the modulation were related to the animals' choice, then the discrimination ability of the observer should have debrain areas.
Table 2. Number of recorded cells

\begin{tabular}{lcc}
\hline & AC & MGB \\
\hline Responsive to $14 \mathrm{kHz}$ & & \\
$\quad$ Tuned to $14 \mathrm{kHz}$ & $114(17 \%)$ & $26(12 \%)$ \\
$\quad$ Tuned to 6 or $31 \mathrm{kHz}$ & $77(12 \%)$ & $31(14 \%)$ \\
$\quad$ Subtotal & $191(29 \%)$ & $57(26 \%)$ \\
Not responsive to $14 \mathrm{kHz}$ & $474(71 \%)$ & $160(74 \%)$ \\
TOTAL & $665(100 \%)$ & $217(100 \%)$ \\
\hline
\end{tabular}
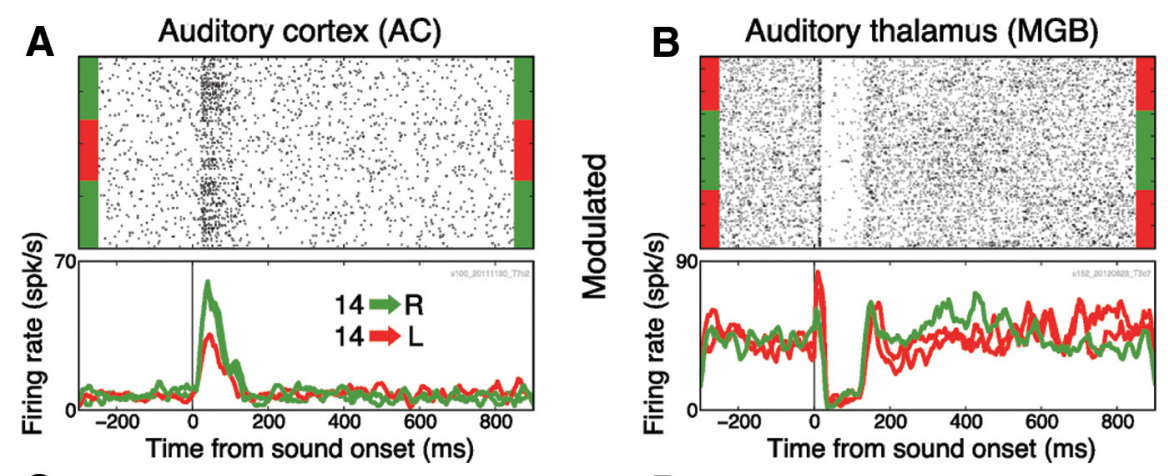

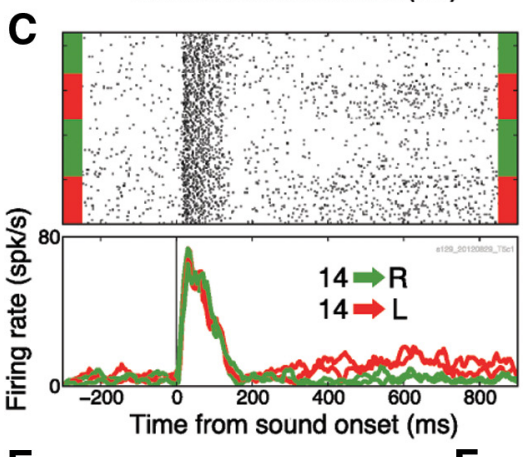

E

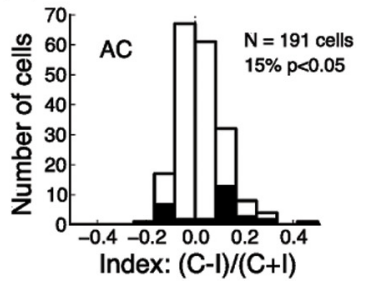

$\mathbf{F}$

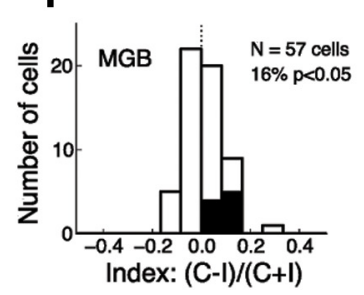

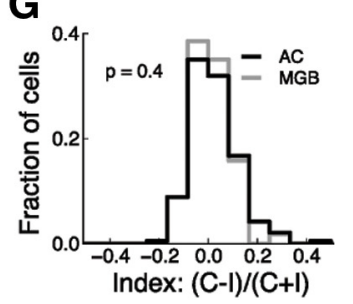

Figure 6. A subset of neurons in thalamus and cortex were sensitive to context. $A$, Responses evoked by the reversing ( $14 \mathrm{kHz})$ sound for a cortical neuron that was sensitive to the meaning of the stimulus. Trials in the raster plot (top) are grouped by the block in which they were presented (three consecutive blocks in this case). The poststimulus time histogram (bottom) shows a clear difference between responses to the $14 \mathrm{kHz}$ sound when it indicated right versus left reward. $\boldsymbol{B}$, Same as $\boldsymbol{A}$, but for a thalamic neuron sensitive to the meaning of the stimulus. In this example, the modulation is most apparent in the onset $(0-50 \mathrm{~ms})$ of the response. C, Evoked responses for a cortical neuron that was not modulated by context. $\boldsymbol{D}$, Evoked responses for a thalamic neuron that was not modulated by context. $\boldsymbol{E}$, The response of $15 \%$ of cortical cells was significantly modulated by context. The histogram shows the modulation index for each cell that was responsive to the reversing $(14 \mathrm{kHz})$ sound. Those in black were significantly modulated ( $p<0.05$, rank-sum test). " $C$ " and "l" are firing rates on trials with reward contralateral or ipsilateral to the recording site, respectively. $\boldsymbol{F}$, Similar to $\boldsymbol{E}$, the response of $16 \%$ of thalamic cells was significantly modulated by context. $\boldsymbol{G}$, Overlaid histograms for cortical $(\boldsymbol{E})$ and thalamic $(\boldsymbol{F})$ cells. There was no difference in the magnitude of modulation between cells from these

creased when trials were swapped. We found that this was not the case in either the AC $(p=0.12 ; n=114)$ or the auditory thalamus $(p=0.23 ; n=26)$. Results were similar when analyzing only neurons with statistically significant modulation in the cortex $(p=0.35 ; n=19)$ or the thalamus $(p=0.86 ; n=7)$. Last, for neurons tuned to the high or low frequency, this hypothesis makes the same prediction as the "adaptive system" hypothesis, which we showed was not consistent with the data. 

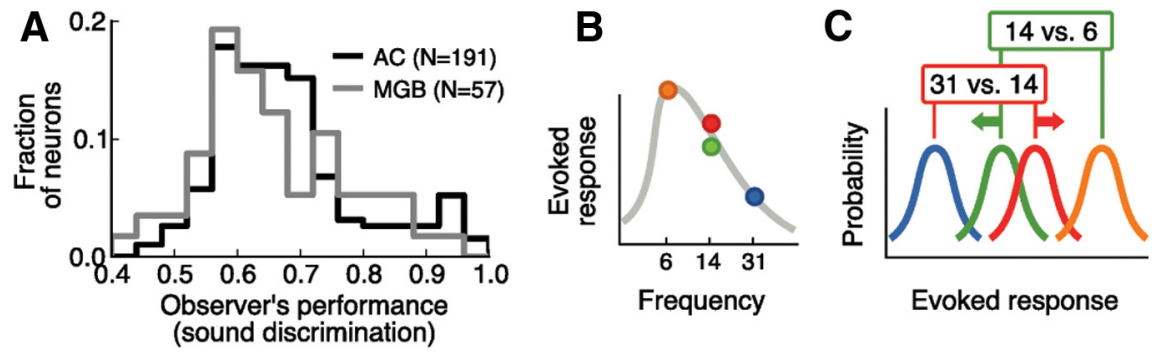

D

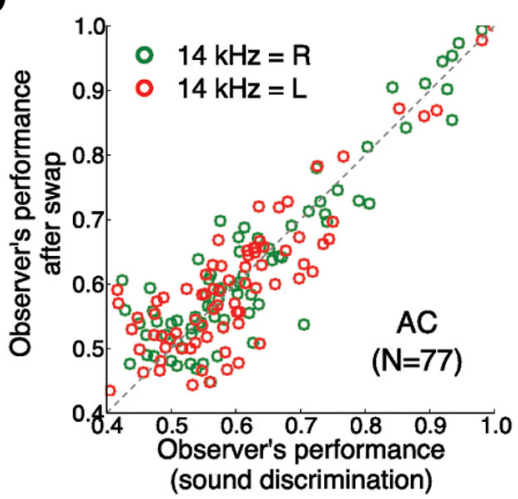

E

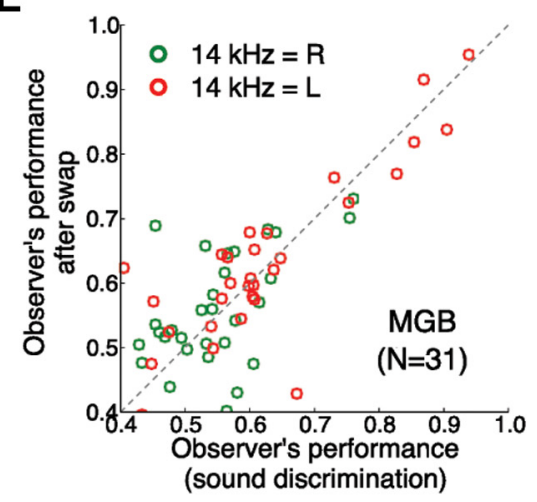

Figure 7. Discriminability of sounds from evoked responses was similar between $A C$ and MGB. $A$, Sound-discrimination performance of an ideal observer for neurons of the $A C$ (black) or the MGB (gray) responsive to the reversing stimulus. Discrimination performance was calculated separately on each of the two contingencies; only the highest of these two values was included in the histogram. The distributions of neuronal performance were indistinguishable between cortical and thalamic cells. $\boldsymbol{B}$, Frequency tuning of a hypothetical neuron with preference for low frequencies. Each dot represents the evoked response for one stimulus in our task (colors as in Fig. 1C). C, Modulation of evoked responses predicted by the "adaptive system" hypothesis for the neuron in $\boldsymbol{B}$. Changes in the response to $14 \mathrm{kHz}$ in the direction indicated by the arrows improve the discriminability of sounds on each contingency ( 31 vs $14 \mathrm{kHz}$ and 14 vs $6 \mathrm{kHz}$ ). D, Effect of swapping trials with $14 \mathrm{kHz}$ stimulus for cortical neurons tuned to high or low frequencies. Each dot corresponds to the observer's performance given the responses of one neuron on each contingency (green: low-boundary; red: high-boundary). If modulation of responses enhanced discriminability, then the performance of the ideal observer should have decreased after swapping trials, yet there was no apparent decrease. $\boldsymbol{E}$, Same as $\boldsymbol{D}$, but for thalamic neurons. There was no decrease in performance after swapping trials.

These results indicate that sound-evoked responses in the AC and the auditory thalamus were not systematically modulated by the choices of the animals.

\section{Discussion}

We trained rats in a flexible categorization task in which the boundary that separates two categories (high- and low-frequency sounds) varied multiple times within a session. Shifts in the category boundary changed stimulus-action associations without changing the associated reward. In this study, we characterized changes in behavioral performance and in the physiological responses of auditory neurons as animals switched between previously learned contingencies. A computational model in which the categorization boundary was represented by synaptic strength of sensorimotor neurons accounted for the main features of behavioral performance. Sound-evoked responses from the majority of cells in auditory thalamus and AC were consistent with signals in the early stages of the model in which sounds are represented independently of their meaning. Notably, we found no differences in the fraction of modulated cells $(\sim 15 \%)$ or the magnitude of this modulation between thalamus and cortex.

\section{Mechanisms for flexible categorization of simple sounds}

We developed a category-learning task in which each of two sound-frequency categories was associated with one reward port. We demonstrated that rats could generalize from a few examples into appropriate categories (Fig. 3) and were able to rapidly adapt after changes in the category definitions (Fig. 2). Our observations suggested a mechanism for category assignment that depended on a subjective category boundary that varied between contingencies. We hypothesized that performance level depended on the distance between stimuli and the boundary and this model accounted for the paradoxical decrease in performance observed for extreme frequencies after a contingency switch (Fig. $2 E$ ). These behavioral changes did not imply a reduction in the overall performance with experience, but instead indicate a change in accuracy for a subset of stimuli, even when these stimuli did not change their associated category. This effect, which to our knowledge has not been discussed in detail previously, will be present in tasks that require difficult discriminations for which perceptual noise becomes relevant.

We accounted for our data using a distance-to-boundary model, yet the underlying neural mechanisms may not necessarily have an explicit representation of this distance. Our study cannot dissociate between different possible mechanisms for the categorization process; for example, similarity to prototypes versus comparison with a boundary versus full representation of the probability distributions of each category (Smits et al., 2006), because any of these theories would be consistent with our data. However, our model provides a biologically plausible example of how to achieve flexible categorization without the need for representing the category boundary in the firing rate of neurons. Moreover, our computational model (Fig. 4) illustrates that noise at the perceptual level, and not at the level of choice execution alone, is required to account for the dynamics of behavior observed in our task. For example, noise in the synaptic connections between cells in the AC and the striatum during behaviors that depend on this neural pathway (Znamenskiy and Zador, 2013) should result in similar performance dynamics to that observed in our task.

What neural mechanisms allow animals to quickly switch between categorization contingencies? We hypothesize two possibilities: (1) changes in associations between sounds and actions are mediated by reward-dependent changes in the synaptic strength of sensorimotor neurons or (2) top-down influences from outside the sensorimotor pathway help reroute sensory signals without long-lasting changes in synaptic efficacy. These ideas correspond to mechanisms hypothesized for learning (Martin et al., 2000; Reynolds et al., 2001) or selective attention (Moore and Armstrong, 2003; Jaramillo and Pearlmutter, 2007), respectively. The computational model shown in Fig. 4 is an implementation of the first idea, but it cannot rule out the second possibility. Further studies are needed to evaluate whether the rapid flexibility required in our task is mediated by long-lasting synaptic changes in the sensorimotor pathway or by top-down influences. 
Influence of context on the physiological responses of auditory neurons

Sound-evoked responses of neurons in the central auditory pathway are influenced by the behavioral context in which sounds are presented (Hubel et al., 1959; Miller et al., 1972; Hocherman et al., 1976. Most studies demonstrating this effect have compared responses under different levels of expectation Jaramillo and Zador, 2011; Ryan et al., 1984), engaged versus passive conditions (Ryan et al., 1984; Otazu et al., 2009; Niwa et al., 2012a), or changes in the reward (Hocherman et al., 1976; David et al., 2012) or punishment (Weinberger, 1993; Fritz et al., 2003) associated with a stimulus. These studies suggest that the magnitude of plasticity is directly related to the magnitude of reinforcement. Our study focused instead on a task in which the amount of reward associated with a sound does not change, but the associated action necessary to collect this reward does change. Our emphasis was on the physiological responses of auditory neurons as animals switched between previously learned contingencies, rather than changes produced by learning an association for the first time.

In a study comparable to ours, Vaadia et al. (1982) evaluated responses of auditory cortical cells during a task that required an overtrained monkey to shift a lever left or right depending on the identity of a sound (tone vs noise). After reversing the associated shift direction, 17\% (23/ 134) of cells showed different responses to a given stimulus, which is consistent with our observations.

Other studies have compared neuronal activity in the AC between correct and incorrect choices (Lemus et al., 2009; Niwa et al., 2012b) using choice probability analysis (Britten et al., 1996). One of these studies (Niwa et al., 2012b) found a small trial-totrial correlation between neural activity and an animal's behavioral choice (choice probability of 0.512 for single units) and a significant modulation of firing rate in $13.8 \%$ (32/232) of cells. Lemus et al. (2009), in contrast, found no significant differences in activity between trials that resulted in hits versus errors.

Neurons from the auditory thalamus also change their responses depending on context, and learning-induced changes have been observed in various subdivisions of the medial geniculate body (Edeline and Weinberger, 1991; Weinberger, 2011). Moreover, studies comparing activity from thalamic cells during engaged versus passive conditions have found changes in spontaneous (Otazu et al., 2009) and evoked (Ryan et al., 1984) firing. In a multisensory task, Komura et al. (2005) found that $14.6 \%$ $(56 / 384)$ of the sound-responsive thalamic neurons changed their firing to the same sound depending on a simultaneous visual cue. When comparing responses between correct and error trials, they found differences close to the response period, but not early during the stimulus presentation. To our knowledge, there has been no comparison of thalamic evoked responses after changes in stimulus-action associations without changes in reward.
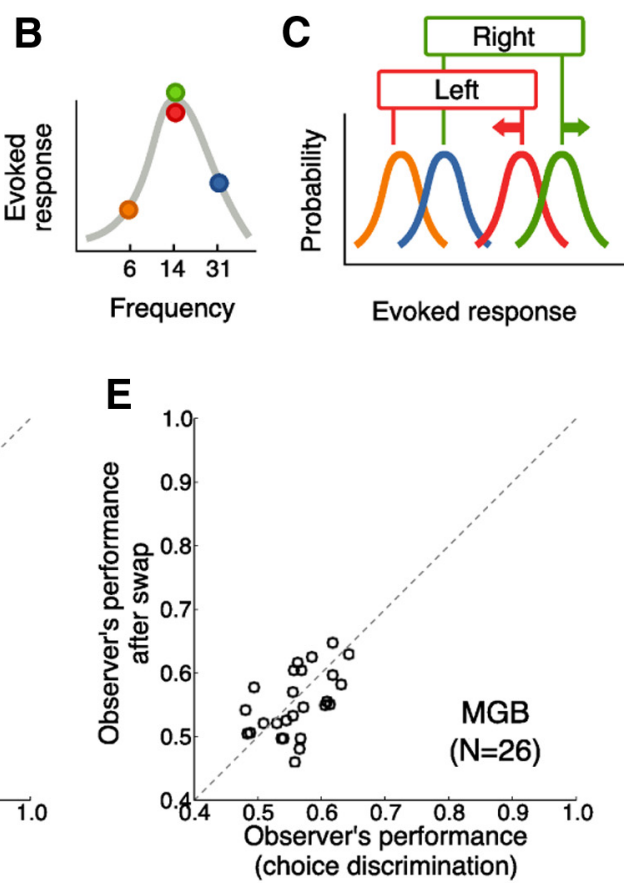

server's performance choice discrimination)

Figure 8. Discriminability of choices from sound-evoked responses. $A$, Choice-discrimination performance of an ideal observer given neurons from the $A C$ (black) or the MGB (gray) responsive to the reversing stimulus. The distributions of neuronal perforand intinguishable between cortical and thalamic cells. $\boldsymbol{B}$, Frequency tuning of a hypothetical neuron with preference the neuron i animal's choice. $\boldsymbol{D}$, Effect of swapping trials with $14 \mathrm{kHz}$ stimulus for cortical neurons tuned to the middle frequency. Each dot 作 but for thalamic neurons. There was no decrease in performance after swapping trials.

The studies presented in the paragraphs above indicate that learning about a stimulus for the first time, or changing the level of engagement in a task, influences the responses of a large fraction of cells in the auditory thalamus and cortex. In contrast, changes in behavioral responses without changes in reward or punishment result in small variations of the sound-evoked activity in only a minority of cells from these brain areas. Our measurements cannot rule out the possibility that plasticity developed in the AC or the MGB as animals were learning to perform the task, given such plasticity in other tasks (Weinberger, 1993; David et al., 2012).

Our study focused on the activity of neurons during stimulus presentation; however, some of these neurons also showed changes in activity to various events within a trial. For example, a subset of cells fired differently depending on the chosen reward port as animals moved to collect reward (observable in Fig. 6C). Studies by others have shown that the firing of auditory neurons in the thalamus and cortex is influenced not only by sounds, but also by rewards and animals' choices (Brosch et al., 2005; Komura et al., 2005; Niwa et al., 2012b). We found indications of similar modulations in both the thalamus and the cortex and a detailed account of these changes will be reported in the future.

Are the effects observed in cortex simply a reflection of effects already present in inputs from the thalamus? Fear-conditioning work by Weinberger (1993) indicated that frequency tuning shifts in the primary AC can be maintained for months, yet shifts in the ventral nucleus of the MGB are transient. Changes in the medial nucleus of the MGB seem to be maintained for longer, but frequency tuning in this nucleus is broader than in A1. It is there- 
fore unclear whether thalamic changes can fully explain changes in the cortex. Our results indicate that evoked responses to simple sounds are similar in thalamus and cortex. This seems to be the case even for natural sounds, and larger changes in information about stimulus identity have been found between the inferior colliculus and thalamus (Weinberger, 1993) than between thalamus and cortex (Chechik et al., 2006). We also found that the same percentage of cells is modulated in auditory thalamus and $\mathrm{AC}$ and that the magnitude of modulation is comparable between the two regions. This leaves open the possibility that flexibility in our task can be mediated by pathways that do not rely on the AC, as has been observed in fear conditioning to simple sounds (Romanski and LeDoux, 1992).

\section{References}

Albanese D, Visintainer R, Merler S, Riccadonna S, Jurman G, Furlanello C (2012) mlpy: Machine Learning Python. Retrieved Feb. 5, 2014, from http://arxiv.org/abs/1202.6548.

Bishop CM (1995) Neural networks for pattern recognition. Oxford: OUP. Britten KH, Newsome WT, Shadlen MN, Celebrini S, Movshon JA (1996) A relationship between behavioral choice and the visual responses of neurons in macaque MT. Vis Neurosci 13:87-100. CrossRef Medline

Brosch M, Selezneva E, Scheich H (2005) Nonauditory events of a behavioral procedure activate auditory cortex of highly trained monkeys. J Neurosci 25:6797-6806. CrossRef Medline

Chechik G, Anderson MJ, Bar-Yosef O, Young ED, Tishby N, Nelken I (2006) Reduction of information redundancy in the ascending auditory pathway. Neuron 51:359-368. CrossRef Medline

David SV, Fritz JB, Shamma SA (2012) Task reward structure shapes rapid receptive field plasticity in auditory cortex. Proc Natl Acad Sci U S A 109:2144-2149. CrossRef Medline

Doron NN, Ledoux JE, Semple MN (2002) Redefining the tonotopic core of rat auditory cortex: physiological evidence for a posterior field. J Comp Neurol 453:345-360. CrossRef Medline

Edeline JM, Weinberger NM (1991) Thalamic short-term plasticity in the auditory system: associative returning of receptive fields in the ventral medial geniculate body. Behav Neurosci 105:618-639. CrossRef Medline

Floresco SB, Zhang Y, Enomoto T (2009) Neural circuits subserving behavioral flexibility and their relevance to schizophrenia. Behav Brain Res 204:396-409. CrossRef Medline

Fritz J, Shamma S, Elhilali M, Klein D (2003) Rapid task-related plasticity of spectrotemporal receptive fields in primary auditory cortex. Nat Neurosci 6:1216-1223. CrossRef Medline

Goldberg TE, Weinberger DR (1988) Probing prefrontal function in schizophrenia with neuropsychological paradigms. Schizophr Bull 14:179-183. CrossRef Medline

Hill EL (2004) Executive dysfunction in autism. Trends Cogn Sci 8:26-32. CrossRef Medline

Hocherman S, Benson DA, Goldstein MH Jr, Heffner HE, Hienz RD (1976) Evoked unit activity in auditory cortex of monkeys performing a selective attention task. Brain Res 117:51-68. CrossRef Medline

Holt LL, Lotto AJ (2010) Speech perception as categorization. Atten Percept Psychophys 72:1218-1227. CrossRef Medline

Hubel DH, Henson C, Rupert A, Galambos R (1959) “Attention” units in the auditory cortex. Science 129:1279-1280. CrossRef Medline

Jaramillo S, Pearlmutter BA (2007) Optimal coding predicts attentional modulation of activity in neural systems. Neural Comput 19:1295-1312. CrossRef Medline

Jaramillo S, Zador AM (2011) The auditory cortex mediates the perceptual effects of acoustic temporal expectation. Nat Neurosci 14:246-251. CrossRef Medline

Kadir SN, Goodman DF, Harris KD (2013) High-dimensional cluster analysis with the Masked EM Algorithm. Retrieved Feb. 5, 2013 from http://arxiv.org/abs/1309.2848.

Komura Y, Tamura R, Uwano T, Nishijo H, Ono T (2005) Auditory thalamus integrates visual inputs into behavioral gains. Nat Neurosci 8:12031209. CrossRef Medline

Lemus L, Hernández A, Romo R (2009) Neural codes for perceptual discrimination of acoustic flutter in the primate auditory cortex. Proc Natl Acad Sci U S A 106:9471-9476. CrossRef Medline

Martin SJ, Grimwood PD, Morris RG (2000) Synaptic plasticity and memory: an evaluation of the hypothesis. Annu Rev Neurosci 23:649-711. CrossRef Medline

Miller JM, Sutton D, Pfingst B, Ryan A, Beaton R, Gourevitch G (1972) Single cell activity in the auditory cortex of rhesus monkeys: behavioral dependency. Science 177:449-451. CrossRef Medline

Moore T, Armstrong KM (2003) Selective gating of visual signals by microstimulation of frontal cortex. Nature 421:370-373. CrossRef Medline

Niwa M, Johnson JS, O'Connor KN, Sutter ML (2012a) Active engagement improves primary auditory cortical neurons' ability to discriminate temporal modulation. J Neurosci 32:9323-9334. CrossRef Medline

Niwa M, Johnson JS, O’Connor KN, Sutter ML (2012b) Activity related to perceptual judgment and action in primary auditory cortex. J Neurosci 32:3193-3210. CrossRef Medline

Ohl FW, Scheich H (2005) Learning-induced plasticity in animal and human auditory cortex. Curr Opin Neurobiol 15:470-477. CrossRef Medline

Otazu GH, Tai LH, Yang Y, Zador AM (2009) Engaging in an auditory task suppresses responses in auditory cortex. Nat Neurosci 12:646-654. CrossRef Medline

Paxinos G, Watson C (2005) The rat brain in stereotaxic coordinates, Ed 5. San Diego: Academic.

Reynolds JN, Hyland BI, Wickens JR (2001) A cellular mechanism of reward-related learning. Nature 413:67-70. CrossRef Medline

Romanski LM, LeDoux JE (1992) Equipotentiality of thalamo-amygdala and thalamo-cortico-amygdala circuits in auditory fear conditioning. J Neurosci 12:4501-4509. Medline

Russ BE, Lee YS, Cohen YE (2007) Neural and behavioral correlates of auditory categorization. Hear Res 229:204-212. CrossRef Medline

Ryan AF, Miller JM, Pfingst BE, Martin GK (1984) Effects of reaction time performance on single-unit activity in the central auditory pathway of the rhesus macaque. J Neurosci 4:298-308. Medline

Smits R, Sereno J, Jongman A (2006) Categorization of sounds. J Exp Psychol Hum Percept Perform 32:733-754. CrossRef Medline

Vaadia E, Gottlieb Y, Abeles M (1982) Single-unit activity related to sensorimotor association in auditory cortex of a monkey. J Neurophysiol 48: 1201-1213. Medline

Weinberger NM (1993) Learning-induced changes of auditory receptive fields. Curr Opin Neurobiol 3:570-577. CrossRef Medline

Weinberger NM (2011) Reconceptualizing the primary auditory cortex: Learning, memory and specific plasticity. In: The auditory cortex (Winer JA, Schreiner CE, eds), pp 465-491. Boston: Springer.

Znamenskiy P, Zador AM (2013) Corticostriatal neurons in auditory cortex drive decisions during auditory discrimination. Nature 497:482-485. CrossRef Medline 